\title{
Oncolytic Virotherapy and Multiple Myeloma
}

\author{
Nahla AM Hamed* \\ Professor of Hematology, Faculty of Medicine, Alexandria University, Egypt
}

Received: September 08, 2017; Published: September 25, 2017

*Corresponding author: Nahla AM Hamed, Professor of Hematology, Faculty of Medicine, Alexandria University, Egypt, Email: drhamedn@hotmail.com

\begin{abstract}
OV immunotherapy is an important emerging cancer treatment modality which uses native or genetically modified viruses to destroy cancers. MM is perhaps the ideal malignancy to target using OVs because of the virus permissivity of neoplastic plasma cells and the diseaseassociated immune paresis. OVs have the potential to mediate both tumor debulking and residual disease elimination avoiding the complex and prolonged current therapy for multiple myeloma.
\end{abstract}

Keywords : OVs: Oncolytic Viruses; MTD: Maximum Tolerated Dose; MM: Multiple Myeloma

\section{Introduction}

OV immunotherapy is a new form of cancer therapy that uses native or genetically modified viruses to selectively enter, replicate and lyse tumor cells without causing harm to normal surrounding tissues [1]. OVs can infect both normal cells and cancer cells, but they demonstrate a preference for cancer cells due to aberrant cell processes and a cancer-induced downregulation of the host antiviral response [2]. Normal cells respond to virus infection by downmodulating their metabolism and/or by undergoing apoptosis, thereby inhibiting virus propagation [3]. OVs also release cancer antigens causing an immune attack against the infected malignant cells [1]. Thus, OVs offer the attractive therapeutic combination of tumor-specific cell lysis together with immune stimulation, therefore acting as potential in situ tumor vaccines [4].

OVs immunotherapy has several features that make it an attractive treatment modality, including the ease of administration, low toxicity profile [1] and potential synergy with other immunotherapeutics such as immune checkpoint inhibitors [1]. They can be readily combined with other agents [4]. A potential advantage of virotherapy is long-term disease control with a single dose [2]. The effectiveness of OVs has been demonstrated in many preclinical studies and recently a major breakthrough for the field in humans, was the US Food and Drug Administration approval of the oncolytic herpes virus talimogene laherparepvec in advanced melanoma [4].

\section{Oncolytic viruses}

Each virus has specific tropism that determines which tissues are preferentially infected. Some viruses have a natural preference for cancer cells [1]. Alternatively, other viruses can be engineered to exploit the defective antiviral defenses of tumor cells [3]. Engineered viruses allows for optimization of tumor selectivity and enhanced immune stimulation [4]. Although several ongoing trials are emphasizing intratumoral delivery of OV, systemic delivery will be absolutely required for treatment of metastatic cancer [1].

Specific targeting of cancer cells for oncolytic virotherapy can be achieved in several ways. Some viruses such as reovirus have a natural preference for cancer cells, whereas other viruses such as measles and Vesicular Stomatitis Virus can be adapted or engineered to make them cancer-specific. Surface markers selectively expressed by tumor cells such as Her2-neu, Prostate Specific Membrane Antigen, and nuclear transcription factors PSA, hTERT, COX-2, osteocalcin can be targeted by using them as receptors for virus entry or as essential cofactors for viral gene expression. An alternative way to 'target' viruses to cancer cells is to selectively eliminate their undesirable tropisms by engineering targets for brain, liver or muscle-specific micro RNAs into their genomes such that the viral life cycle is selectively blocked in the relevant target tissue [3].

OVs can kill infected cancer cells in many different ways, ranging from direct virus-mediated cytotoxicity to immune-mediated cell death [1]. Conventional concepts of cell death (apoptosis, necrosis or autophagy) are inadequate to fully describe the complex cell killing scenarios encountered in virotherapy. The OV typically takes over and controls the molecular cell death machinery of the infected cancer cell, allowing death to occur only after available cellular resources have been maximally exploited for the synthesis and assembly of new viruses. In addition, OV can mediate killing of uninfected cancer cells by indirect mechanisms such as destruction 
of tumor blood vessels, amplification of specific anticancer immune responses or through the specific activities of transgene-encoded proteins expressed from engineered viruses [3].

\section{Virotherapy of Multiple Myeloma}

Because of the virus permissivity of neoplastic plasma cells and the disease-associated immune paresis, MM is perhaps the ideal malignancy to target using OVs. Current therapy for MM is complex and prolonged. Antimyeloma drugs are combined in induction; consolidation and/or maintenance protocols to destroy bulky disease, then suppress or eradicate residual disease. OVs have the potential to mediate both tumor debulking and residual disease elimination [5]. Virotherapy is an emerging approach in the early stages of clinical development for MM. Trials emphasis on a subset of myeloma patients who have not been too heavily pretreated and still have a strong immune response. Several viruses are being exploided for virotherapy of MM, but most remain in pre clinical development including adenovirus, vaccinia virus, herpes simplex virus type-1, myxoma virus and coxsackie virus A21. Several viruses have reached clinical investigation for the treatment of MM, including reovirus, measles virus, and vesicular Stomatitis virus [4].

The measle virus was evaluated in a phase $1 / 2$ trial of patients with recurrent or refractory MM. Two of measles seronegative patients experience response but one patient remains in complete remission for 4 years. Both responding patients did not have detectable antimeasles antibodies. Adverse effects include fever, severe headache, tachycardia and hypotension during the initial hours of infusion [2]. A phase 1 study of reovirus among patients with relapsed stable MM resulted in stable disease in $42 \%$ of patients. the MTD was not reached. In an effort to improve the outcomes with reovirus, a phase $1 \mathrm{~b}$ trial is ongoing in which reovirus is combined with bortezomib and dexamethasone [4]. A new virus study was opened in April 2017 with the vesicular stomatitis virus [2].

Further investigations are required to overcome antiviral response immunity that can limit the efficacy of this therapeutic strategy [6]. Clinical trials using cellular carriers as Trojan horses to more efficiently deliver the virus to sites of myeloma growth is especially important for patients who have antimeasles antibodies from childhood vaccination or infection [2]. OVs may meet criteria as ideal purging agents for hematological malignancies such as MM and leukemia cells. Specifically, certain OVs selectively target malignant hematopoietic cells while sparing normal hematopoietic stem and progenitors. This capacity to purge autologous HCT grafts makes OVs particularly attractive for potential use in the clinical transplant setting. A few OVs have already been translated into the clinic [7].

Future advances in oncolytic virotherapy include identification of drugs that accelerate intratumoral virus propagation, new strategies to maximize the immunotherapeutic potential of oncolytic virotherapy, and clinical confirmation of a critical viremic thereshold for vascular delivery and intratumoral virus replication [3].

\section{Conclusion}

Better understanding of the use of OV immunotherapy in myeloma patients alone or in combination is still needed.

\section{References}

1. Babiker HM, Riaz IB, Husnain M, Borad MJ (2017) Oncolytic virotherapy including Rigvir and standard therapies in malignant melanoma. Oncolytic Virotherapy 6: 11-18.

2. Blevins Primeau AS. Virotherapy for multiple myeloma: the current state of research. Available at www.cancertherapyadvisor.com/ multiple-myeloma/virotherapy- multiple-myeloma-current-treatmentlandscape.

3. Russell SJ, Peng KW, Bell JC (2014) Oncolytic virotherapy. Nat Biotechnol 30(7): 658-670.

4. Lawler SE, Speranza MC, Cho CF, Chiocca EA (2017) Oncolytic viruses in cancer treatment: A review. JAMA Oncol 3(6): 841-849.

5. Naik S, Nace R, Federspiel MJ, Barber GN, Peng KW, et al. (2012) Curative one-shot systemic virotherapy in murine myeloma. Leukemia 26(8): 1870-1878.

6. Oliva S, Gambella M, Boccadoro M, Bringhen S (2017) Systemic virotherapy for multiple myeloma. Expert Opinion on Biological Therapy 10: 1-3.

7. Bais S, Bartee E, Rahman MM, McFadden G, Cogle CR (2012) Oncolytic virotherapy for hematological malignancies. Advances in Virology 2012(2012): 1-8.

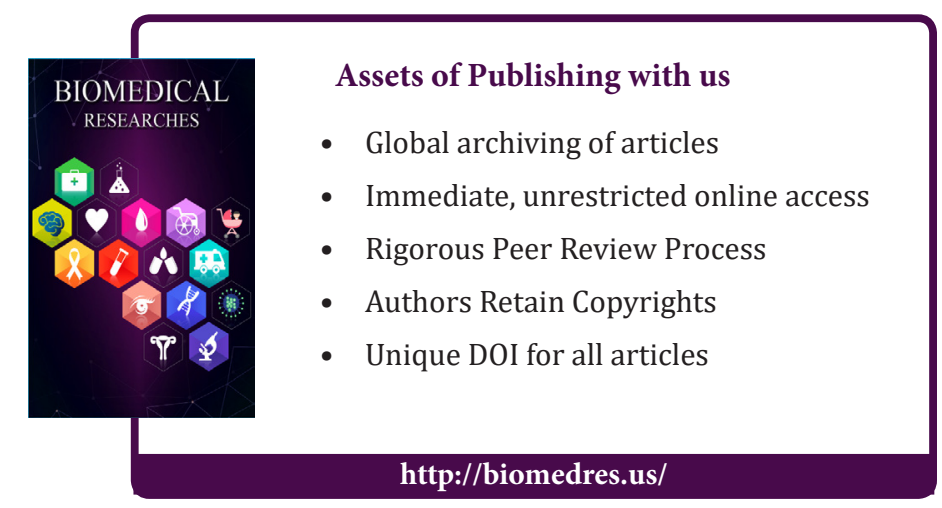

